\title{
A Journey of Digital Co-Production: Introducing the "E-Nabling Digital Co-Production" Framework and Its Performance in Dementia Research
}

Mauricio Molinari-Ulate ( $\square$ mmolinari@ides.es )

Psycho-Sciences Research Group, Institute of Biomedical Research of Salamanca, University of

Salamanca

Rebecca Woodcock

NIHR MindTech MedTech Co-operative

Isabelle Smith

NIHR MindTech MedTech Co-operative

Henriëtte G. van der Roest

Department on Aging, Netherlands Institute of Mental Health and Addiction, Trimbos Institute

Manuel A. Franco-Martín

Psycho-Sciences Research Group, Institute of Biomedical Research of Salamanca, University of

Salamanca

Michael P. Craven

NIHR MindTech MedTech Co-operative

\section{Research Article}

Keywords: Dementia, COVID-19, patient and public involvement, PPI, dementia research

Posted Date: February 28th, 2022

DOI: https://doi.org/10.21203/rs.3.rs-1363528/v1

License: (a) (1) This work is licensed under a Creative Commons Attribution 4.0 International License.

Read Full License 


\section{Abstract \\ Background}

The rapid transition to digital working, accelerated due to the response to the COVID-19 pandemic, has impacted the involvement of patients and public in research. For this reason, this paper presents a narrative of a co-production journey experienced by a Participant and Public Involvement (PPI) group since the lockdowns and recommendations regarding digital working in England, followed by a practical implementation of a framework within the context of dementia research.

\section{Methods}

Three online workshops were performed with a group of researchers, facilitators and contributors with experience in PPI in dementia research, and a dementia PPI group that kept working during the pandemic. Thematic analysis was chosen for the data analysis. The project was targeted as a PPI activity and the MindTech Involvement Team (PPI group) was involved in the entire process.

\section{Results}

After a refinement of the coding structure, the MindTech Involvement Team and Project Leaders identified the potential of using four coding areas resulting in the 'E-nabling Digital Co-production' Framework. Through this framework, conflicting positions were identified associated with the transition to digital working. Two main remarks were shared by the participating groups regarding e-PPl; the wider potential in terms of non-geographical constraints, and the perspective of more business-like sessions with reduce social interactions and communication. Specifically for dementia research, e-PPI may allow participants to attend more meetings, although the mutually supportive environment of face-to-face meetings is diminished, and carers can miss out on respite opportunities.

\section{Conclusions}

This public engagement activity introduced the 'E-nabling Digital Co-production' Framework showing an initial potential for the analysis of e-PPI flaws and benefits. It was possible to identify insights of e-PPI regarding general concerns and specific to dementia research. However, the most significant contribution of the framework is the potential to support local journeys of co-production in ongoing public involvement activities.

\section{Plain English Summary}

The COVID-19 pandemic has greatly impacted the engagement of patient and public participants in research. Lockdowns, social distancing, and reduced physical contact have affected the involvement of 
experts by experience in research studies. In particular, the pandemic has triggered a rapid transition to digital working, increasing the use of Information and Communication Technologies such as video conferencing on computers and mobile devices. There was little time to reflect on the consequences of digital working in PPI. This project tells the journey of digital co-production of an active PPI group during the pandemic. It highlights the challenges and potential for e-PPI (electronic/digital PPI) which was implemented in the context of dementia research. It includes an initial response of different stakeholders to the transition to digital working and resulted in a co-produced framework for researchers, PPI professionals and public contributors.

\section{Background}

Participant and Public Involvement (PPI) has gained more attention in recent years across all areas of health research (1), and dementia is not an exception $(2,3)$. For this reason, it is considered as a cornerstone for governmental and ethical policies in health research along with the development of PPI best practice guidelines $(2,4,5)$. It has been defined as a research project or public policy development carried out with or by patients or members of the public that is beyond their engagement as subjects (57).

Due to its practical benefits that enhance the quality of the research (3-5), PPI has become more a requirement than a research approach option, including funding applications for health research (5). Whilst democratic rationales $(8,9)$ may receive less attention than technocratic or transactional motivations, patient involvement has the potential to either address or exacerbate existing inequalities within health outcomes (10). Indeed, these existing inequalities risk being further compounded through the COVID-19 pandemic (11).

However, since the beginning of the pandemic, declared by the World Health Organization in March 2020, the involvement of patients and the public in research has been challenged because of social distancing, lockdowns, and other reduced physical contact (12). Therefore, quick responses and adjustments have been needed, enhancing the implementation of Information and Communication Technologies (ICT) (13, 14). Moreover, this may have exacerbated issues already faced and gaining increasing prominence within both PPI and wider research participation regarding a lack of diversity within patients and public (15). With the definition, breadth and theoretical underpinning of PPI already conceptually challenged and contested (8) alongside a range of practices and values underpinning its delivery (16), the move to digital represents a further domain in which complicated dynamics exist.

For this reason, this paper first introduces a narrative, describing a journey of co-production as experienced by one PPI group (MindTech Involvement Team) within the context of a rapid and enforced transition to digital working. Following on from this and facilitated by the co-production process itself, the discussion moves to e-PPI within the context of dementia research, developing both best practice guidance through this newly developed framework, before considering the potential for its wider application, including those under-served by health research (17). 


\section{Co-producing an immediate local response:}

The move to digital PPI (referred to henceforth as e-PPI) methods will be explored initially through the lens of one PPI group based within MindTech, a national centre focussing on the development, adoption, and evaluation of new technologies for mental healthcare and dementia. The MindTech Involvement Team, comprised of people bringing their own lived experiences of mental health as well as expertise in the processes of patient and public involvement, occupies a strategic and advisory role, with the aim of involving the public in all aspects of research across the organisation.

Experiencing an immediate and instrumental shift to digital PPI in March 2020, the MindTech Involvement Team, shared the experience of other PPI groups not previously operating online involvement methods and continued to meet regularly during the pandemic. Although adapting their practice to enable continued involvement, PPI members, PPI staff and researchers acknowledged the challenges and opportunities that this brought to ensuring continued meaningful involvement. Furthermore, understanding how these differ at an individual, group and research level, informed a desire to understand more about the opportunities and challenges ushered in by a new era of digital involvement. This led to a local response at the MindTech Involvement Team and at organisational level, co-producing an overview of the primary areas that were impacted by the shift to e-PPI and the local actions taken to both mitigate challenges and harness the potential from these new ways of working. Originally presented at the MindTech Symposium in December 2020, an initial response to the transition to e-PPI through ICT was proposed (Additional file 1. Overview of Digital Considerations)

From developing an initial generic set of considerations regarding e-PPI, this continued with an e-Project conceived in November 2020 as part of the first author's (MMU) training programme placement for the European DISTINCT network (https://www.dementiadistinct.com/). Working in collaboration with MindTech, this afforded the opportunity for further involvement and application of these considerations within the context of dementia-specific PPI. Through an iterative process and with the view to creating a systematic and potentially more widely applicable framework, this resulted in the development of the 'Enabling digital co-production" model (Fig. 1) for researchers, PPI professionals and PPI public contributors to explore the challenges and potential for e-PPI.

A study of the move to e-PPI in relation to an evolving framework may serve to enhance understanding of the use of ICT for PPI, not only in dementia research or during COVID-19, but also regarding other constraints that might diminish public and patient involvement; for example, access to rural and remote areas, limited availability of services and health professionals, distance from healthcare services, poverty, PPI representatives' health conditions, such as fatigue, mobility restrictions, work or family commitments, limited budget for research with PPI, etc. $(6,7,18)$.

\section{Dementia and PPI}

Whilst PPI groups nationally grappled with many similar elements, systematic consideration and understanding of the relative successes of different PPI groups within a transition to e-PPI remained 
elusive. It was proposed that although their remained a set of shared considerations, success may be predicated on both the area of health research and significantly on the type of group experiencing the transition to online involvement.

People Living with Dementia (PLwD) and carers represent one such group. Although far from homogenous, PPI groups working with PLwD may share a set of shared challenges and opportunities with respect to this digital transition and which are additional to considerations already well articulated (2). With ICT (telephone or e-mail) already identified as a valuable tool for PPI representatives' engagement in dementia research (6), further insights may be made through exploring the experiences of using ICT, with a view to identifying better approaches for public involvement and making the most of experts by experience.

\section{Methods}

\section{Developing a Dementia e-PPI Project}

Continuing the focus on co-production, the MindTech Involvement Team participated in three sessions with project leaders at subsequent stages, with one member participating as a co-lead of the project and involved as a facilitator of the online workshops. Members of the Involvement Team contributed from the outset and throughout all stages, supporting the project design, development, delivery, and synthesis of the outcomes from the online workshops.

\section{Online workshops}

Three online workshops were conducted and chaired by the project leaders ( $M M U$ and BW) and the CoLead from the MindTech Involvement Team (IS). During the workshops, facilitators provided two roles: a) facilitation of the workshops and discussion; b) administrative and inclusion role, with a person in charge of taking field notes and checking the chat box. Field notes were chosen to gather the information as they have been previously implemented in similar public engagement projects $(19,20)$ and because verbatim transcripts would not be available as the sessions were not recorded to maintain the policies of the PPI groups involved and as this project was organised as a PPI activity (21). The online platform used was Microsoft Teams (MS Teams) as this was the tool facilitated by the institutions involved.

Targeted as a PPI activity, the project team selected a workshop approach as opposed to alternatives such as semi-structured interviews with individual PPI members, considering it the most pragmatic strategy to working online with PPI groups. Use of a familiar working methodology, where the public contributor could feel comfortable participating was also considered, with the PPI group included for this project already familiar with taking part in online group discussions.

Workshop formats allow an exchange of ideas within a scaffolded structure, inclusion of potential challenges or allow for a range of positions expressed within a supported environment, thereby enabling various positions within a group forum to be identified. By undertaking workshops online, it is also 
possible to share comments through the chat function, where a parallel discussion can be facilitated, allowing people to share their thoughts without having to speak to the rest of the group.

Before each of the sessions, participants were provided with a project information sheet and a semistructured guide of possible topics and questions to cover at the discussions. A one-minute pre-recorded pitch was shown at the beginning of each of the sessions by way of introducing the project, inviting individuals to participate, and as an "ice-breaking" strategy to initiate the activity. The time for the sessions varied from 25 minutes to approximately an hour.

\section{Groups involved and settings}

To develop a broader perspective on the challenges and experiences related to the transition to e-PPI, researchers, PPI coordinators and facilitators, and PPI members were targeted and included in the set of workshops.

Two groups were contacted and agreed to participate, consisting of a) a group of researchers, facilitators, and contributors of PPI groups with experience in dementia research, and b) the "Dementia, Frail Older People and Palliative Care Patient and Public Involvement Advisory Group" (the Advisory Group from now on) from the University of Nottingham. Using existing PPI groups provides safe and structured settings for working with PLwD and carers, recognising the need for increased attention to ethical and welfare issues, already documented as requiring additional thought within the context of PPI for PLwD (2).

\section{Qualitative analysis method}

A thematic analysis was the chosen approach for the analysis of the results. In keeping with a methodological approach based on co-production, members of the MindTech Involvement Team and Project Leaders started coding the information, identifying the potential to utilise the Overview of Digital Considerations (Additional file 1) to support this endeavour. Although providing limited value in supporting these initial coding attempts, it quickly emerged that with further refinement, there was potential to utilise this approach further. After initially reviewing with MindTech Involvement Team members, two of the Project Leads (BW and IS) proposed that consolidation into four key areas may support categorisation of contributions resulting from workshop discussions. After this first iteration, the final Project Lead joined the analysis, confirming the possibility of using the framework as a coding tool (MMU). Overarching categories were suggested, with consolidation into four main areas, with the inclusion of the concept of 'involvementability' as identified within the Researcher and PPI Staff Workshop and resulting in the 'E-nabling Digital Co-production' Framework (Fig. 1).

\section{'E-nabling Digital Co-production” Framework}

The framework targets four key areas:

\section{a) Technological:}

Understanding the technological landscape that digital PPI is operating within, including consideration of the constraints, preferences, and opportunities that technology can provide, is an important prerequisite 
to effective digital collaboration. Decision-making regarding technological platforms was often completed early on within the transition to digital PPI, without full understanding of the range of potential digital tools for meeting and online collaboration.

Technological considerations can be seen as instrumental in determining future success yet may have been afforded only limited initial consideration and co-production. Whilst shared decision-making regarding the use of technology, including functional and operational components can be considered essential for facilitating effective engagement, many aspects remain determined by organisational constraints and without the input of public contributors. As a category, it is proposed that technological considerations are revisited regularly, recognising the fast pace of developments in online collaborative platforms and potential for growing confidence in using these alternative methods, amongst both researchers, PPI staff and public contributors. This is identified as the first area within the framework, acknowledging the need to co-produce a response to digital PPI in an evolving landscape.

b) Resources: Digital involvement may require additional resources. Understanding this category as including a broad concept of resources, including the potential for emotional resilience as well as financial or time implications, it is essential that where additional requirements are necessary, that this is planned from the outset. As well as additional facilitation roles, onboarding sessions and the cost of coproduction platforms themselves, these could include phone credit/printing etc./software/budgeting for an increased frequency but shorter meetings. Wider resources may be understood as the need for additional training for researchers, PPI staff and public contributors to support the use of new technology.

\section{c) "Involvementability"}

The concept of 'involvementability' first emerged as part of discussions with Researchers and PPI practitioners, specifically within the context of understanding how e-PPI is impacted by the field of health research. Within translational research and with digital health interventions (DHIs) in particular, the processes for obtaining PPI input may be complicated by digital delivery methods for patient and public involvement. Use of offline prototypes or the ability for researchers to support and observe public contributors as they experience and navigate newly developed technologies may be limited; the ease of 'involvement' being impacted by the interaction between method and subject. Involvementability is perhaps best viewed as an example of a non-functional requirements, a concept that aims to describe requirements that are related to the success of a design task or process but are not integral to its content (22).

The 'E-nabling Digital Co-production' Framework adopts the concept of non-functional requirements, proposing that the nature of involvement methods interacts with the demographic and the nature of research itself, leading to varying success. By addressing questions, such as how codesign methods may differ in a digital space or how the area of health research itself may impact on the extent that involvement can easily translate to a digital space, it seeks to extend our understanding of the nuances within process, practice, product, and population.

\section{d) Ethical and Welfare:}


Whilst ethical and welfare considerations are likely to have been considered early on within the transition to digital PPI methods, the breadth and range of issues within this category are extensive. PPI groups already addressing these as part of previous practice, are compelled to revisit and review how digital PPI interacts with a range of areas, including: welfare; digital exclusion; impact of digital engagement on social communication; power; safeguarding; privacy, confidentiality and data security.

\section{Results}

Three sessions on three different days were undertaken in June 2021. Five researchers and/or facilitators with experience in PPI in dementia research participated in the workshop; while in the Advisory Group workshop, eleven people with various experiences as public contributors, attended the meeting. An additional individual meeting was conducted, as one researcher was not able to attend the group session. A total of 14 pages of notes were gathered from the workshops, from a total time of two hours and two minutes of meetings.

In order to explore the move to e-PPI for dementia research during the pandemic, the insights from the workshops were mapped against the four areas of the 'E-nabling Digital Co-production' Framework, as it was refined following the co-production journey described previously. This approach also allowed for the opportunity to identify and highlight specific insights for dementia research.

The following section demonstrates how insights were then categorised according to the co-produced framework areas, and which itself, represents a potentially significant output emerging from this work. In general, positive and negative aspects were expressed by the public contributors, for this reason the results will be separated according to these subcategories. Also, dementia specific remarks will be highlighted separately under each area.

\section{a) Technological}

All remarks about technology aspects came from the researchers and facilitators group. Three main technical issues were highlighted: online platform alternatives, technical support, and accessibility. Concerning access to different platforms, facilitators/researchers, commented on restrictions due to institutional rules or policies which determine the platform options, often requiring use of MS Teams. Consequently, use of alternative platforms was not straightforward. Significantly, a range of alternative platforms were identified as potentially having an impact on solving various barriers or constraints to participation, with some individuals more familiar with or preferring other platforms.

Other reflections emphasised the need for a technical or administrative support with responsibility during virtual meetings for helping to resolve technical issues, and the concern about difficulties identified in giving full access to other participants.

\section{b) Resources}


A diversity of topics arose concerning this area. On the positive side, there was no need for the usual time allocated to making arrangements in terms of venues, catering, or other coordination, such as transportation for PPI representatives. However, it is understood that other resource requirements may be needed instead, such as the time for additional support staff to facilitate online delivery, or the practice of providing additional reimbursement to recompense costs incurred through online working. Another positive was the reflection that virtual meetings were more focussed or "straight to the point" although this may itself contribute to some of the perceived lack of informal communication and connections that face-to-face PPI may create. Also mentioned was that online meetings allowed members to attend more meetings. Finally, both researchers/facilitators and the members of the PPI group, considered that e-PPI had a wider potential reach, with virtual meetings enabling connections with researchers or participants that are geographically dispersed. In terms of resource considerations this represents a positive, however, from an ethical perspective that will be considered shortly, this may exacerbate existing inequalities by reducing inclusion of those individuals or communities not already involved within health research. On the negative side, attendees underlined that planning e-PPI is more time consuming, and others mentioned that controlling time and contributions could be harder.

Particularly for carers of PLwD, it was considered that virtual meetings helped to overcome some of the limitations related to their care role, such as concerns around time away, finding a solution for care, or other time constraints, as they could attend the PPI sessions from home.

\section{c) Involvementability}

Three main barriers for e-PPI involvement stood out regarding this area under the following subcategories: virtual meeting limitations, communication, and social interaction.

Virtual meeting limitations: researchers and facilitators emphasised the limitations of the type of activities that could be done; some of the insights referred to the difficulties in involving individuals when different devices are required. One example was the need for multiple devices as in the case of using a laptop for ICT while the research was to evaluate another device or software, such as an app on a phone or tablet. Another issue arising from the use of video communications, mentioned by researchers, is the limited view of physical prompts or other non-verbal communication.

Communication: both groups considered that communication is less effective during virtual meetings. Reflections that reinforced this idea related to feedback mechanisms and interchanges between attendees and researchers that were missing or diminished in an online exchange. It was proposed that this was affected by the reduced non-verbal communication, resulting in less fluid discussion (e.g., more formal turn-taking) and not being able to see all participants on the screen at the same time. However, a positive reflection from researchers/facilitators was that virtual meetings served to encourage reflection about communication methods, particularly the role of raising hands and waiting for an opportunity to participate. 
One area of potential ambiguity, as to the positive or negative impact of e-PPI on participation, was whether this method served to increase the inclusion of those less confident in participating. Whilst participation could be more easily regulated with facilitation leading to greater inclusion, it was also highlighted that online interactions may create or reinforce additional barriers to engagement.

Specifically for dementia research, participants underlined the need to consider the cognitive abilities as the level of attention or concentration needed for virtual meetings could affect the discussion and engagement, for example, someone may forget their contribution by the time they have an opportunity to talk.

Social interaction: overall participants had experienced less social interaction through virtual meetings. They mentioned a decrease of informal social interaction, such as breaks during the sessions that allow spontaneous conversations and interchange between attendees, and lack of opportunities to share and meet with others, leading to a more "business-like" meetings.

In terms of positive contributions, researchers and facilitators considered that e-PPI could be less threatening concerning the physical social interaction and that, normally, the individual will be participating in a safe environment (e.g., their home). Additionally, they mentioned that people wishing to isolate for any reason (e.g., COVID) can be included, and that close and strong relationships could be developed.

Regarding dementia specific remarks, it was considered that meeting online may diminish the opportunity of a respite and supportive space for members providing care, which seems to be accomplished in faceto-face meetings.

\section{d) Ethical and welfare}

The following three main topics were identified in this area:

Diversity and inclusion: as a negative perception, researchers and facilitators considered that e-PPI could be a barrier for inclusion as the group of individuals attending virtual meetings may stay the same without new members coming along. Also, they considered that the group does not represent all sectors of the community.

On the contrary, virtual environments could offer the opportunity to include those that have not been considered for several circumstances. However, it was noted that to achieve this, recruitment methods would need to be improved with further recognition that this is compounded, with increased difficulties in recruiting PLwD in an online context.

Digital inclusion: several barriers were identified by members from the Advisory Group, including that with e-PPI, some individuals could feel that they are not part of the research team as the sense of group is missing, and that difficulties with the technology or the dislike for virtual meetings was a factor in losing participants. A similar barrier was considered by researchers and facilitators as they mentioned that e-PPI 
could be excluding individuals that lack the skills and confidence needed to use the technology, which might be exacerbated in vulnerable populations, and that even those familiar with the platforms struggle when technical issues occurred.

Furthermore, equity was arising as a concern in terms of the technology use and support, particularly because some individuals could have better access while others do not (e.g., good bandwidth) and those in need of support or living alone might not be able to join (e.g., PLwD).

Regarding dementia specific remarks, the participation and presence of the caregiver is harder to control in virtual meetings, which might lead to a diminished participation of PLwD.

Ethical issues: some of the barriers mentioned by researchers and facilitators related to the need for clarity regarding reimbursement of public contributors, informed consent to record virtual meetings, and emotional support when people get distressed or frustrated.

Specifically for dementia, the severity of cognitive impairment arose as a consideration. As cognitive function determined the level of support needed at the virtual meetings, this was not always straightforward to assess or support remotely. Furthermore, it was identified that there was an increased difficulty in controlling levels of caregiver support and input, with potential to diminish participation from the individual living with dementia.

\section{Tips to improve e-PPI}

By using the 'E-nabling Digital Co-production' Framework, a number of recommendations emerged to improve virtual meetings for e-PPI. These have been extracted from the workshops' discussions and are summarised in Fig. 2.

\section{Discussion}

\section{A new framework}

The project aimed to consider digital PPI in a dementia-specific context and as a by-product, led to refining existing guidance and co-producing the 'E-nabling Digital Co-production' Framework, introduced here as a tool for researchers and PPI coordinators to identify and discuss challenges and opportunities provided by e-PPI. In this regard, the framework was found as a useful potential tool for analysis of the information, as demonstrated in the results section. Offering a step forward for thematic analysis, its four areas served as the main codes, with insights from the participating groups mapped accordingly. Whilst not initially available for the analysis, the journey to co-produce the final framework represents a key narrative component and emerging output.

Although the four areas were used as the final codes, the framework is flexible enough to allow the inclusion of subcategories without modifying its content, meaning, and structure. For example, under the 
Ethical and Welfare code, three subcodes were identified (Diversity and Inclusion, Digital Inclusion, Ethical Issues) and all of them are under the definition for this category.

Another relevant characteristic of the framework is its potential to adapt to the specific context of the target population. Even though it was developed as a response to the transition to digital co-production (e-PPI) due to the pandemic, without targeting a particular PPI area, this project shows that it can be used for specific contexts, in this case for dementia research. It is proposed that the inherent flexibility lends itself to be used with other populations and contexts involving digital PPI. Also, its adaptability to other situations makes it a suitable tool to study how the digitalization of the PPI meetings has impacted the involvement and engagement of the public in research, even more so in the COVID-19 context.

Likewise, the framework is not exclusive to e-PPI, rather a tool with the potential to consider how PPI is approached, both considering the current pandemic conditions, and going forward as a facilitator for PPI groups to explore their own preferences and the implications of different models of PPI within the postpandemic transition. The experience of online meetings and increased familiarisation with digital platforms may serve to accelerate the acquisition of technological skills amongst both academic and patient populations.

Regarding our findings in terms of the challenges and approaches of e-PPI, it is possible to identify both positive and negative opinions concerning digital co-production. However, ambiguities were also highlighted, with conflicting opinions between the public contributors during the workshops that opened deeper discussions, and where a clear position in terms of pros and cons was not obvious.

As an example, e-PPI was considered as a barrier in terms of the diversity and inclusion of the PPI groups as the same members are always attending the sessions, however, it was also mentioned that the virtuality offers the opportunity to include others that have not been considered for various circumstances. Similarly, within the dementia context, e-PPI seems to offer to carers the opportunity or the capacity to attend more meetings but simultaneously they may lose a potentially valued respite or time away from caring, as they do not have to transfer to the venue for the meeting.

Such ambiguity extends across different areas. This is the case with communication, where both groups agreed that e-PPI acts as a barrier for communication and social interaction compared to experiences of meeting face-to-face. This led to more business-like sessions with a decrease in social experiences and support, as well as reduced non-verbal communication. Nonetheless, it offers an opportunity to reevaluate the importance of meeting etiquette and communication styles, such as taking turns, which also can offer an opportunity (or a barrier) for those less self-confident in participating. Equally, technical functions of platforms potentially allow for asynchronous and anonymous contributions, both of which could support wider involvement.

Indeed, such ambiguity leaves one aspect clearer; e-PPI has both potentials and challenges that must be evaluated as part of a context specific and co-produced response. Even with a retreat from the pandemic, 
e-PPI will remain embedded as a potential method to add value to existing approaches or to be considered as part of an evolving hybrid toolkit to perform PPI in research.

Despite ambiguity and conflicting positions, two remarks were shared by the participating groups and could be considered as the main potential and main barrier of e-PPI according to our results. On the positive side, people identified the wider potential of e-PPI in terms of non-geographical constraints, leading to the possibility of participation and inclusion of people from anywhere. In terms of resource considerations this represents a positive. However, from an ethical perspective, that will be considered shortly, this may exacerbate existing inequalities by reducing inclusion of those individuals or communities not already involved within health research. The transition to digital e-PPI has not occurred in a vacuum, with other external drivers and the recognition that diversity and inclusion within research is an area that requires multi-stakeholder action and commitment $(1,14)$. Such topical debate dissects discussion around digital exclusion and wider inclusion and arguably increases the need for PPI practitioners to explore the nuances of how e-PPI impacts this debate.

To increase social interaction, e-PPI methods could be adapted to include other approaches, potentially offering increased opportunity for this social interchange between public contributors and researchers and facilitators. This is the case of forum initiatives which could be more conducive to recreating the informal spaces of face-to-face meetings and overcome this vision of business-like sessions. This may further uncover ambiguities surrounding the purpose of PPI and the role of reciprocity in establishing relationships, where it is accepted that public contributors engage in health research with numerous and varying motivations. Just as the literature on volunteering explores this phenomenon (23) it should be both recognised and reflected in efforts to reframe digital e-PPI that can mitigate the perception of a reduction to a transactional exchange, that has been highlighted.

New ethical challenges are ushered in within the digital domain, particularly the digital divide in populations, as the familiarity and access to the technologies remains inequitable, leading to an exclusion of vulnerable populations and some sectors of the society $(14,24)$. Also, administrative considerations such as the consent form for recording or the reimbursement for public contributors, are topics that need more discussion. This associates with other considerations that whilst not arising during these workshops, such as the security, password use or management of the data and which are all relevant concerns due to the progress of the new technologies.

The framework also offered possibilities through insights collating recommendations to improve e-PPI, which were shown earlier in Fig. 2. As was seen in the figure, tips are also mapped according to the areas of the framework, and they were obtained from the comments from researchers, facilitators, and members of the Advisory Group. As was reflected in the results, most of the insights overlapped between both workshops' groups, suggesting that the results presented in this project are shared by the different populations involved in the different workshops. However, further work exploring the subtleties and prioritisation by participants and organisers of e-PPI, may serve to uncover further areas to improve and consciously address the potential for exacerbating existing power balances through online practices. 


\section{Dementia specific}

Lastly, we were interested to see if the framework could target specific insights for e-PPI in dementia research. Some of the remarks seem to be relevant for carers in general, regardless of the condition of the person being cared for, for example, on the positive side e-PPI seems to allow attending more sessions without being worried or stop attending due to their care role. However, by meeting online, virtual public contributors are missing a respite and supportive space that is present in face-to-face meetings, also they might be excluding those living alone or needing more support, and it seems harder to control the presence of the caregiver which can diminish the individual participation.

Nonetheless, specifically for the dementia context, the severity of the cognitive impairment is something to be aware of during e-PPI meetings as it might affect the engagement and participation of individuals. Particularly, there are some concerns regarding the cognitive abilities needed for e-PPI, as it was considered that the level of attention and concentration is different compared to face-to-face meetings, also memory impairments could affect the access to the platform (e.g., forgetting the password or the steps to joined in) or the contribution to the discussion as while waiting for the turn to talk, someone could forget the idea. Additionally, the level of impairment determines the level of support needed, which leads to the need of training, an additional person supporting the participants, the inclusion of informal carers, or other relevant potential solutions.

\section{Limitations and further projects}

The project was conceived as a public engagement activity and our purpose was to make a first attempt on introducing the 'E-nabling Digital Co-production' Framework, for this reason, the number of participants is not considered as a representative sample, nor did we explicitly collect demographic information which is usual practice in PPI (21). Furthermore, our sample does not involve those PLwD who were currently not undertaking PPI digitally, naturally limiting our ability to understand the experiences of those not participating in PPI currently. Therefore, results should be used as an insight to improve future approaches to e-PPI in dementia research and other related contexts.

Due to the COVID-19 pandemic, we experienced difficulties with finding active PPI groups of PLwD, including those struggling to meet online. This led us to contact a convenience sample of PPI groups know by the project team to be active at that time, therefore, the only group that we could include for this project was the Advisory Group. However, it is mainly integrated by PLwD informal caregivers, so the participation of PLwD for this project is missing. It is also worth noting that this group had existing experience of online working. Furthermore, the time to perform the workshop with this group was fewer than the workshop done with researchers and facilitators, which could have affected the volume and depth of insights identified from its members.

Additionally, due to the online methodology used in this project, the public contributors and researchers/facilitators may be biased to those who are more comfortable with online tools. Also, only one digital platform was used (MS Teams), as it was the only one available according to the institutions 
involved. This was also brought during the workshops as one of the main limitations of e-PPI, as institutions normally offered only one option which limits the engagement of public contributors as they might not be familiarized with the same digital platform. Future studies could contribute by controlling the familiarity with online tools and by adding and comparing different digital platforms.

Likewise, it remains that some of the advantages of a workshop format were not fully realised within an online context and conversely, through undertaking these sessions online, advantages such as access to alternative method of communicating via chat functions, represented a potential advantage. Indeed, it is recognised as a methodological limitation that there is an element of self-selection, through working with groups that already had experience of operating within an online environment. Although field notes are not the most complete means to collect data for qualitative analysis, this methodology has been successfully used in other public engagement projects $(19,20)$. Due to the circumstances already mentioned regarding the pandemic, the policies of the PPI groups involved, and because this project was a PPI activity, verbatim transcripts of the workshops were not gathered (21).

As the 'E-nabling Digital Co-production' Framework seems to be a suitable tool to target issues and opportunities of e-PPI, future public involvement activities or research projects could test it and use it to improve its usefulness. The flexibility that it offers could be used in several contexts broaden its usability.

It could be beneficial for researchers, organisations and individuals undertaking future activities within PPI to utilise the 'E-nabling Digital Co-production' Framework, due to the holistic nature of the themes present that can give a strong understanding of how to most effectively work with a group of participants. Regarding dementia digital PPI, it would be beneficial to undertake further research on a larger scale and possibly incorporate comparisons between types of dementia to explore if this has an impact on preferences. Furthermore, this framework could be used on a micro level such as a local PPI group with individual social and health needs to better understand the digital preferences of the group and consequently have better outcomes for sessions. On the other hand, the framework could be utilised on a macro level to undertake national studies to understand the PPI digital working needs of those with certain health conditions. Importantly, as the stages of the pandemic change and restrictions are lifted and face-to-face working is resumed, it is imperative that those facilitating PPI activity are aware of the impact that digital PPI working has had upon the preferences among participants they plan to work with.

Finally, with potentially increased ability to facilitate face-to-face PPI activity, future research regarding digital PPI could include those not currently involved in digital working for a broader understanding. Overall, it would be recommended future research continues to explore digital PPI in both dementia and other conditions to gain a clearer understanding of how we can better facilitate future digital PPI working in these ever-changing times.

\section{Conclusion}

The 'E-nabling Digital Co-production' Framework has been introduced through this public engagement activity, showing an initial usefulness for researchers, facilitators, and members from the public to 
understand and analyse issues and opportunities regarding e-PPI. The framework was not only able to map insights regarding general concerns of digital PPI, but also allowed for identifying specific insights for dementia research. While potentially a useful approach that could be generalised to further projects and public involvement activities, most significantly, is the contribution to supporting a local journey of co-production, as took place in its development.

\section{Abbreviations}

COVID-19

Coronavirus disease 2019

PPI

Participant and Public Involvement

e-PPI

electronic/digital Participant and Public Involvement

ICT

Information and Communication Technologies

DISTINCT

Dementia:Intersectorial Strategy for Training and Innovation for Current Technology

PLwD

People Living with Dementia

MS Teams

Microsoft Teams

DHIs

Digital Health Interventions.

\section{Declarations}

\section{Ethics Approval}

Advice was sought from the Chair of the University of Nottingham Faculty of Medicine and Health Science Research Ethics Committee (REC). It was considered that REC approval to conduct this project was not required as it is a public engagement activity with all participants contributing as experts (REC correspondence reference no. 255-0521).

\section{Consent for publication}

Not applicable

\section{Availability of data and materials}

Data sharing is not applicable to this article as no datasets were generated or analyzed during the current study. 


\section{Competing interests}

The authors declared that they have no competing interests.

\section{Funding}

This project has received funding funding form the European Union's Horizon 2020, as part of the Marie Skłodowska Curie Actions - Innovative Training Network, H2020-MSCA-ITN (under grant agreement number 813196). The work was supported by the NIHR MindTech MedTech Co-operative and NIHR Nottingham Biomedical Research Centre. The views expressed are those of the authors and not necessarily those of the NHS, the NIHR, or the Department of Health and Social Care.

\section{Authors' contributions}

MMU, executive researcher of the project and wrote the first draft of the manuscript. MMU, RW and IS substantially contributed to the acquisition, analysis, and interpretation of data. MMU, RW, IS and MC substantially contributed to the study design. All authors substantially contributed to the conception of the work and the final revised version of the manuscript. All authors have approved the submitted version that involved the author's contribution to the study and have agreed both to be personally accountable for the author's own contributions and to ensure that questions related to the accuracy or integrity of any part of the work, even ones in which the author was not personally involved, are appropriately investigated, resolved, and the resolution documented in the literature.

\section{Acknowledgements}

Special thanks are due to the members of the MindTech Involvement Team who were engagedi during the different stages of the project, to the group of researchers, facilitators, and contributors with experience in dementia research, and the University of Nottingham Dementia, Frail Older and Palliative Care, Patient and Public Involvement Advisory Group which supports a wide range of research projects undertaken in the Faculty of Medicine and Health Sciences.

\section{References}

1. National Institute of Health Research. National Standards for public involvement in research. 2018.

2. Gove D, Diaz-Ponce A, Georges J, Moniz-Cook E, Mountain G, Chattat R, et al. Alzheimer Europe's position on involving people with dementia in research through PPI (patient and public involvement). Aging Ment Health. 2018;22(6):723-9.

3. Miah J, Dawes P, Edwards S, Leroi I, Starling B, Parsons S. Patient and public involvement in dementia research in the European Union: a scoping review. BMC Geriatr. 2019;19(1):220.

4. Charlesworth G. Public and patient involvement in dementia research: Time to reflect? Dementia (London). 2018;17(8):1064-7. 
5. INVOLVE. Briefing notes for researchers: public involvement in NHS, public health and social care research. Eastleigh: 2012.

6. Burton $\mathrm{A}$, Ogden $\mathrm{M}$, Cooper $\mathrm{C}$. Planning and enabling meaningful patient and public involvement in dementia research. Curr Opin Psychiatry. 2019;32(6):557-62.

7. Dogba MJ, Dossa AR, Breton E, Gandonou-Migan R. Using information and communication technologies to involve patients and the public in health education in rural and remote areas: a scoping review. BMC Health Serv Res. 2019;19(1):128.

8. Ives J, Damery S, Redwod S. PPI, paradoxes and Plato: who's sailing the ship? J Med Ethics. 2013;39(3):181-5.

9. Williams O, Sarre S, Papoulias SC, Knowles S, Robert G, Beresford P, et al. Lost in the shadows: reflections on the dark side of co-production. Health Research Policy and Systems. 2020;18(1):43.

10. Madden M, Speed E. Beware Zombies and Unicorns: Toward Critical Patient and Public Involvement in Health Research in a Neoliberal Context. 2017;2(7).

11. Bambra C, Riordan R, Ford J, Matthews F. The COVID-19 pandemic and health inequalities. J Epidemiol Community Health. 2020;74(11):964-8.

12. NHS Health Research Authority. Public involvement in a pandemic: Lessons from the UK COVID-19 public involvement matching service. 2021.

13. Johnson F. Health information professionals: delivering core services and value in extraordinary times. Health Info Libr J. 2020;37(3):245-7.

14. Cadel L, Marcinow M, Sandercock J, Dowedoff P, Guilcher SJT, Maybee A, et al. A scoping review of patient engagement activities during COVID-19: More consultation, less partnership. PLOS ONE. 2021;16(9):e0257880.

15. Ekezie W, Routen A, Denegri S, Khunti K. Patient and public involvement for ethnic minority research: an urgent need for improvement. 2021;114(7):347-50.

16. Gradinger F, Britten N, Wyatt K, Froggatt K, Gibson A, Jacoby A, et al. Values associated with public involvement in health and social care research: a narrative review. Health expectations: an international journal of public participation in health care and health policy. 2015;18(5):661-75.

17. Yu Z, Kowalkowski J, Roll AE, Lor M. Engaging Underrepresented Communities in Health Research: Lessons Learned. Western journal of nursing research. 2021;43(10):915-23.

18. Brighton LJ, Pask S, Benalia H, Bailey S, Sumerfield M, Witt J, et al. Taking patient and public involvement online: qualitative evaluation of an online forum for palliative care and rehabilitation research. Res Involv Engagem. 2018;4:14.

19. Craven MP, Goodwin R, Rawsthorne M, Butler D, Waddingham P, Brown S, et al. Try to see it my way: exploring the co-design of visual presentations of wellbeing through a workshop process. Perspectives in Public Health. 2019;139(3):153-61.

20. Harrington K, Craven MP, Wilson ML, Landowska A. Exploring User Expectations of Brain-Training and Coaching Technologies for Cognitive Health. Human-Computer Interaction Human Values and 
Quality of Life. Lecture Notes in Computer Science. 2020; p. 49-60.

21. Hoddinott P, Pollock A, O'Cathain A, Boyer I, Taylor J, MacDonald C, et al. How to incorporate patient and public perspectives into the design and conduct of research [version 1; peer review: 3 approved, 2 approved with reservations]. 2018;7(752).

22. Craven MP, Lang AR, Martin JL, editors. Developing mHealth Apps with Researchers: MultiStakeholder Design Considerations. In: Design, User Experience, and Usability User Experience Design for Everyday Life Applications and Services. Cham: Springer International Publishing; 2014. p.15-24.

23. Kee YH, Li C, Wang JCK, Kailani MIB. Motivations for Volunteering and Its Associations with Time Perspectives and Life Satisfaction: A Latent Profile Approach. Psychological Reports. 2017;121(5):932-51.

24. Amann J, Sleigh J. Too Vulnerable to Involve? Challenges of Engaging Vulnerable Groups in the Coproduction of Public Services through Research. International Journal of Public Administration. 2021;44(9):715-27.

\section{Figures}




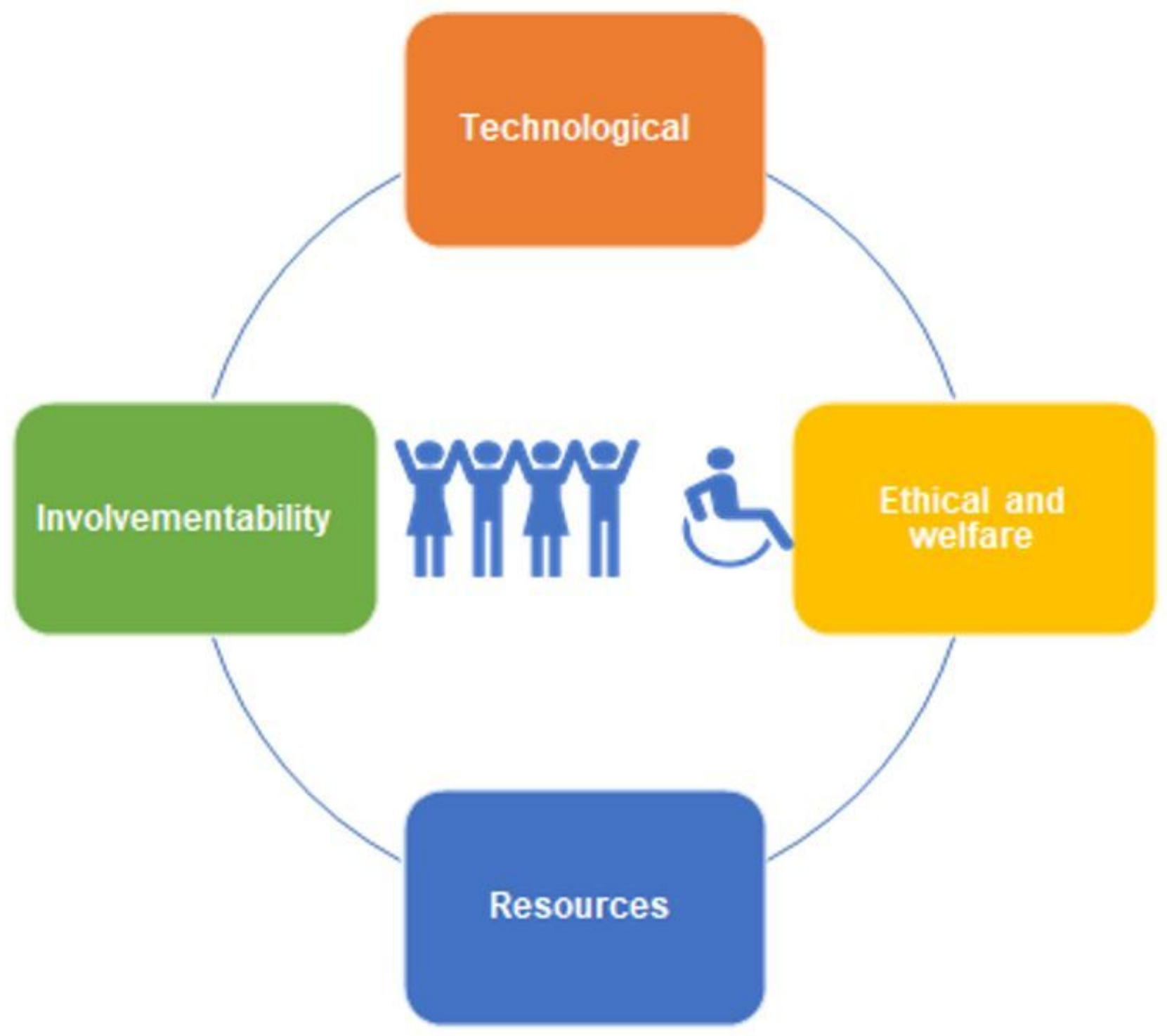

Figure 1

“E-nabling Digital Co-production” Framework 


\begin{tabular}{|c|c|c|}
\hline Technological & "Involvementability" & Resources \\
\hline $\begin{array}{l}\text { - Virtual platforms should } \\
\text { be considered as part of } \\
\text { the toolkit to perform } \\
\text { PPI. } \\
\text { - More familiarity and time } \\
\text { using technology might } \\
\text { improve usability with the } \\
\text { platforms. } \\
\text { - Other options could be } \\
\text { social media, telephone } \\
\text { or websites, however, } \\
\text { control on who is } \\
\text { participating might be } \\
\text { lost. } \\
\text { - A hybrid option was } \\
\text { consideredas a } \\
\text { possibility. People meet } \\
\text { face-to-face but could } \\
\text { allow other to join } \\
\text { remotely. }\end{array}$ & $\begin{array}{l}\text { - Smaller groups could } \\
\text { improve communication } \\
\text { as less people show on } \\
\text { screen and there are less } \\
\text { people talking. } \\
\text { - Individual sessions or } \\
\text { smaller groups might } \\
\text { help with attention } \\
\text { wandering and make } \\
\text { people feel more } \\
\text { comfortable. } \\
\text { - Bringing ideas or } \\
\text { activities (ice-breakers) to } \\
\text { prompt participation and } \\
\text { group cohesion. }\end{array}$ & $\begin{array}{l}\text { - Organisers need to make } \\
\text { sure additional resources } \\
\text { are built into the PPI } \\
\text { support and are } \\
\text { budgeted for (e.g., } \\
\text { technical support staff, } \\
\text { additional reimbursement } \\
\text { to recompense costs } \\
\text { incurred through online } \\
\text { working). } \\
\text { - Split sessions might be } \\
\text { useful. E.g., have a short } \\
\text { onboarding session for } \\
\text { new participants and then } \\
\text { follow-on with the actual } \\
\text { PPI session. } \\
\text { - Face-to-face training } \\
\text { sessions could help } \\
\text { participants learn how } \\
\text { to use platform. }\end{array}$ \\
\hline
\end{tabular}

Ethical and welfare

- Provide the opporunity to decide between face-toface, virtual meetings, or hybrid option, when possible. Do not make assumptions about the group members' preferences.

- Consider the configuration of the group (e.g., history of the group location, health conditions, etc.) when deciding which platform to use.

- Follow-up around welfare.

\title{
Figure 2
}

Tips to improve e-PPI meetings

\section{Supplementary Files}

This is a list of supplementary files associated with this preprint. Click to download.

\author{
- Additionalfile1.docx
}

